\title{
PRELIMINARY EXPERIMENTS ON WRITING REACTIONS.
}

\author{
BY FRANK N. FREEMAN, M.A. \\ Assistant in Psychology, Yale University.
}

This paper reports a method of recording the movement made during the drawing of a line by means of a pencil as in writing. From this record the details of the form of the movement, the rate of the movement, and the changes in pressure which accompany the movement can be examined with great exactness. The measurements here reported are preliminary to an examination of different forms of writing and individual variations in writing movements. They give the time involved in starting simple and complex movements of different forms, and the time required to stop a yertical or circular movement when these are under way. In general it was found that stopping a movement involves more complex adjustments than starting, and when the final form of the movement is complex the time of starting is appreciably lengthened.

\section{Apparatus.}

The apparatus used in this experiment was devised for the purpose of making a general investigation of the writing coordination. The investigation has reached a stage which justifies a preliminary report giving a description of the apparatus, together with some account of experiments on the fundamental modes of reaction involved in writing, namely reaction by stopping, starting, and changing the direction of movements made with a pencil.

The method is essentially a modification of the kymograph method. A strip of paper, $B$, Fig. 76 , travels across the writing surface, $A$, on which the reactions are made. As a moving strip is not a convenient surface upon which to write, a typewriter ribbon is placed above the moving strip and above the ribbon is a fixed sheet of paper on which the reactor writes. Thus, when the subject writes on the fixed sheet, the moving strip underneath takes the record from the ribbon as though the strip were being written upon directly, and at the same time the subject has the record of what he is writing before him as usual and is in no way discommoded by the movement of the 


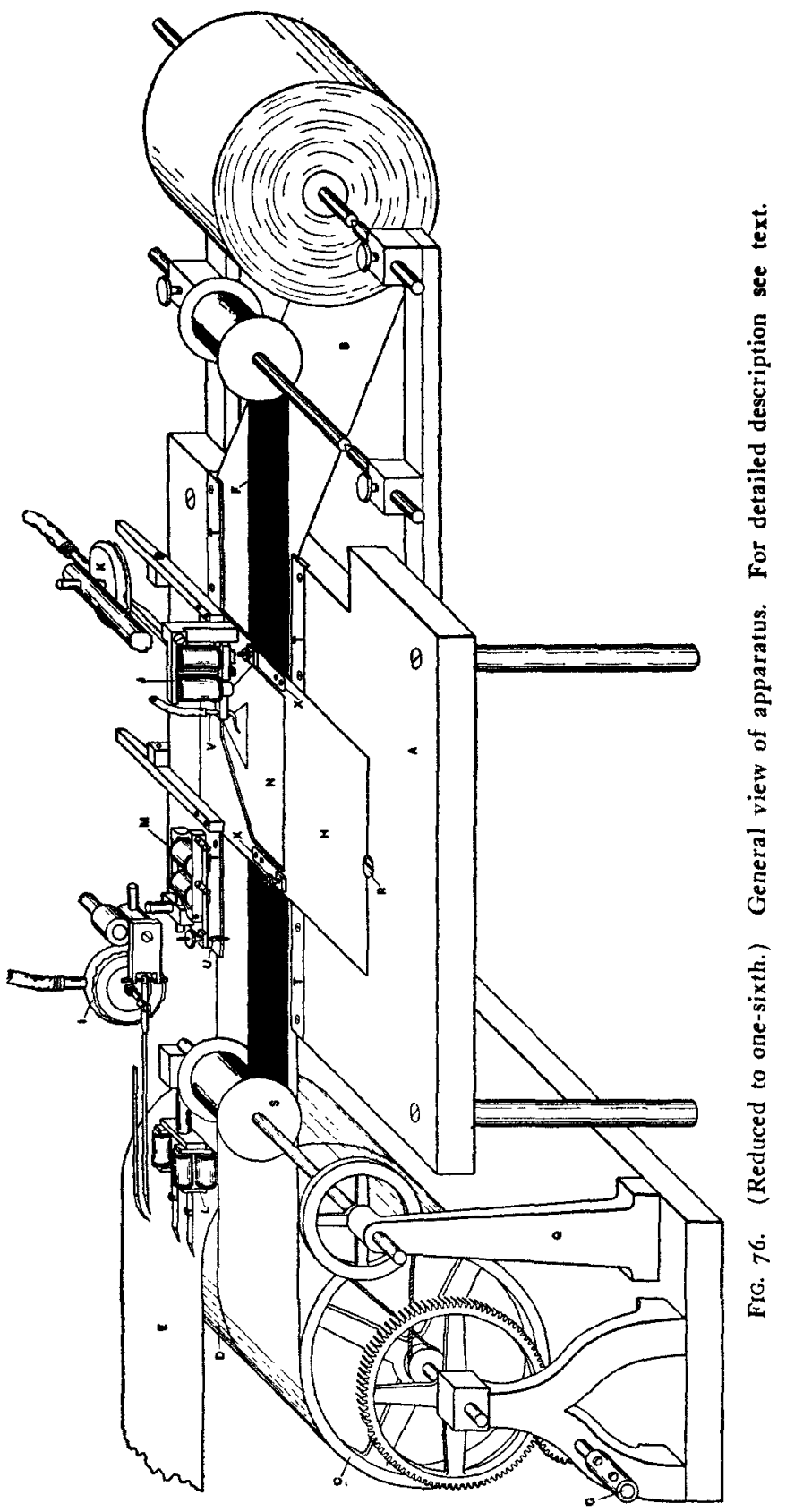


strip underneath. The record on the upper sheet we may call the primary record and the record on the moving strip the secondary record. The sheet of paper and the strip, on which the primary and secondary records respectively are taken, will be referred to as the primary sheet and the moving strip. A sheet of carbon paper was first used for the transfer of the record, but it was found that the carbon soon wore off, especially when the pencil was held in one place for any length of time, and the ribbon moving at a slow rate was substituted.

Fig. 76 gives a general view of the apparatus. Two bars extending from the metal base $A$ support the roll of paper and the spool of ribbon from which the strips $B$ and $F$ are unrolled. $B$ and $F$ pass across plate $A$ to the drum $C$ and the spool $S$, the latter being supported by the post $Q$ and one not shown in the figure. The drum and spool are driven through spur gear connections by the shaft $G$, which is in turn connected with a driving shaft. The apparatus is coupled into the driving shaft and uncoupled by a friction clutch of the type shown in Fig. 97, page 37I, of this volume. The apparatus can thus be set in motion at full speed, and it can also be instantly set free when the record is complete. The motion thus obtained is very regular and easy to control. The motion of the typewriter ribbon spool is greatly reduced from that of the drum, with the shaft of which it is connected by a belt.

In order to hold in position the primary sheet of paper on which the reactor writes and to support the hand, a plate, $H$, is placed over the primary sheet. A rectangular opening, $N$, is made in this plate to expose a writing surface on the primary sheet. The plate, $H$, is hinged at the back of the main base by two bars, so that it may be raised to insert the paper. Fig. 77 shows it raised from the base. Two small pins, $O, O$, pierce the primary sheet of paper and fit in the hole $R$, and one not shown in the figure, and keep the primary sheet from slipping when the strip and ribbon pass beneath it. These pins are above and below the moving strip. In order to get an even writing surface the plate is set into the main base, so as to lie flush with the general surface, and is held down by a screw, $R$, Fig. 76 . The moving strip of paper and ribbon are also 
set below the surface in a channel which is cut in the main base. Two guides, $T, T$, on each side of the moving strip keep it straight. The upper ones are slightly adjustable, so as to suit minor differences in the width of the paper.

In order to obtain a record of the relative position of the primary sheet and the moving strip, two pencil points are set through holes in the hinged plate, $H$. These pencil points make two dots upon the primary sheet and two lines on the strip. The points are shown in Fig. $76, X, X$, and the holes through which they project in Fig. $77, E, E$. The points are set on two flat springs and are adjustable with screws, so that they may be set against the paper with varying degrees of pressure.

Since the speed of movement of the strip is not perfectly uniform, an electric marker writing tenths of seconds, $J$, Fig. 76 , is pivoted to a post set on the hinged plate, $H$, and is adjusted by a screw so as to bring the writing point against the paper. It writes through an opening in the primary sheet upon the moving strip beneath. In order to keep the primary sheet from blotting this line, it is held up from the moving strip by two small brass clips, $T$, Fig. 77 , and the time line passes between these clips.

The glass pen, $V$, Fig. 76, which is used for the time record, is a form of capillary pen. To prevent clogging and uneven flowing, the opening in the point is made fairly large and the flow of ink controlled by a regulating air chamber. The upper end of the glass tube is inserted in a rubber tube which allows the point to move freely, and the tube is connected with a tambour. The rubber head of this tambour can be raised or lowered by a screw, and the ink thus made to flow slower or faster.

In order that the relation of the reactor's record to the time line might be easily determined, the reactor was required in this experiment to make all movements holding the pencil against guides which were placed in a known relation to the time marker. These guides can be set in the opening, $N$. One is a straight strip of brass which is a guide for the vertical line and the other a circular piece of brass which is a guide for the circle. The circular guide is about $4.5 \mathrm{~cm}$. in diameter. 
In order to get a record of the time when the signal was given in the reaction experiment, a second marker, $M$, Fig. 76 , was clamped to the writing base and made to write on the moving strip. This marker is $14.5 \mathrm{~cm}$. from the first marker, $J$, and by making a correction of this distance in reading the record, it can be correlated with the time marker and the traced record. This marker writes with a special form of pen.

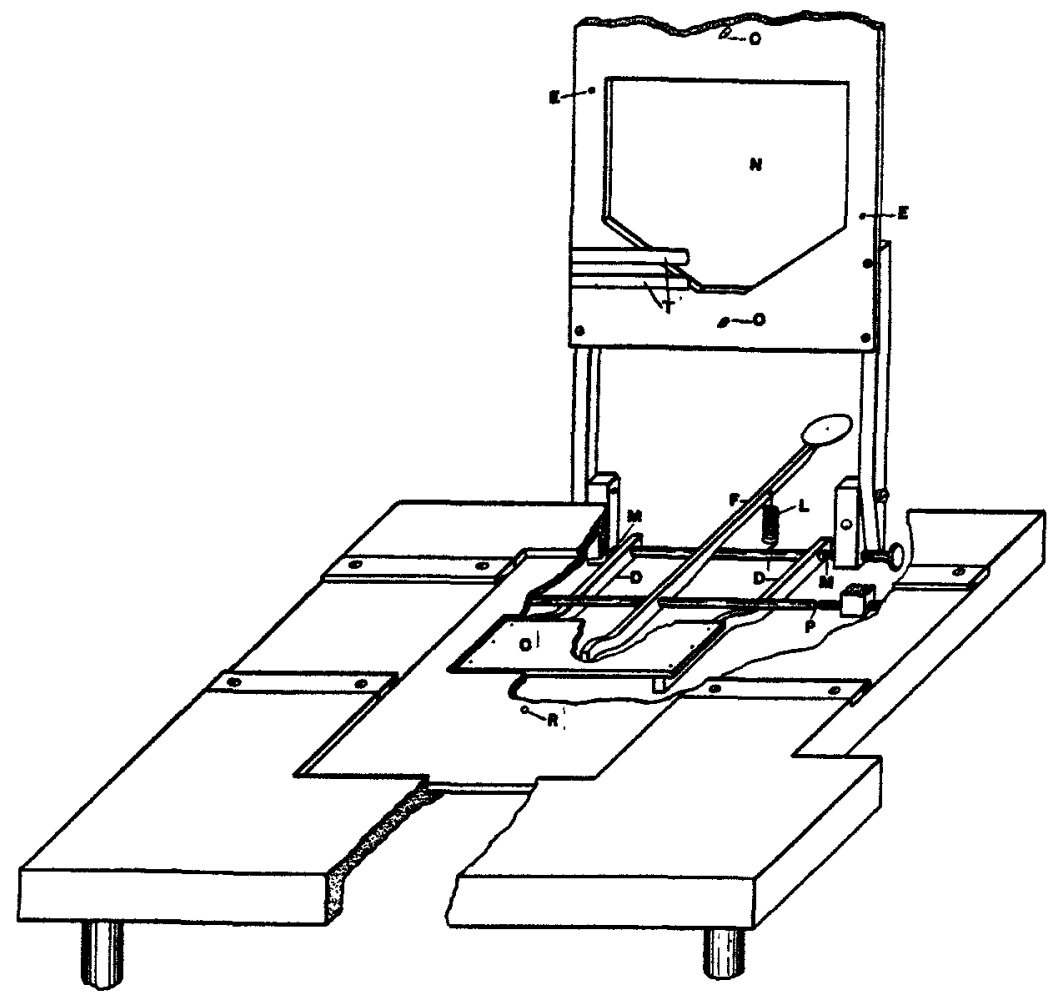

Fig. 77. (Reduced to one-fifth.) Supplementary view of apparatus.

This pen, $U$, Fig. 76 , is made from a conical piece of steel by drilling a hole through the base and cutting a slit from this hole to the point. The marker is in circuit with a telegraph sounder which gives the stimulus for the reaction. Both marker and sounder are operated by a mercury key, thus giving on the moving strip with the other records the time of occurrence of the sound to which the subject is to react. 
Besides the speed of the reactor's movements, it is desirable that the variations in the pressure of the pencil against the paper should also be recorded. The arrangement for securing a record of the pressure is shown in Fig. 77. Under the paper upon which the reactor writes is a small table $C$, set into an opening in the base. The opening in which this table is set is situated immediately below the opening, $N$, of the hinged plate, so that the table occupies all of the writing space. The table is capable of an upward and downward movement, for it is fixed to the two bars, $D, D$, which are in turn fixed to the axis working in the pivot joints, $M, M$. The radius of movement of the table is, accordingly, the length of the bars $D, D$, or I 7 $\mathrm{cm}$., and the direction of movement during a slight displacement is practically in a vertical line. The extent of movement of the table is magnified five times by means of the lever, $F$, which has its fulcrum at $P$. A disk on the outer end of this lever is in contact with the rubber of the tambour $K$, Fig. 76 . The inner end of the lever, which is rounded, bears up against the table, making a sliding contact. In order to lessen the weight and consequent inertia of these parts, the table and its connections are made of aluminum. The long arm of the lever nearly balances the weight of the short arm together with the table and its supporting bars. The slight residue is counterbalanced by a light spring, $L$. This can be adjusted so that it will bring the table quickly back to position, but will not prevent a delicate response of the lever to a very light presssure on the table. The spring, $L$, as well as the tambour, with which a disk on the end of the lever is in light contact, are supported by a rod, shown in Fig. 76, fastened to the main base. The tambour is adjustable so that its head will just touch the lever when the table is in position. This apparatus responds with delicacy sufficient to easily record all the ordinary changes in pressure during writing. Tests with weights show that it will record changes in pressure of from 20 to 300 grams.

The remainder of the apparatus for recording pressure is shown in Fig. 76. The receiving tambour, $K$, is connected with the recording tambour $I$, which writes on a long strip of smoked paper, $E$. This strip travels over the drum, $D$, and another 
drum 3.5 meters away. The drum $D$ is clamped by an adjustable screw to the same shaft as the drum $C$, so that both of them can be driven together, or either one can be run separately by loosening the screw which clamps $D$ to the shaft. Above the tambour pointer is a fixed pointer which traces a straight line with which to compare the pressure curve. The pressure curve is correlated with the speed curve on the moving strip, $B$, by means of one pointer of the double marker, $L$, which is in circuit with the marker, $J$, on strip $B$. The time of the reaction signal is also recorded on this smoked record by the other pointer of the marker, $L$, in the same circuit with the sounder.

To sum up then, by means of the apparatus above described, one can obtain two records, one showing all the details of the rate, and the other all the details of pressure in any writing or drawing movement. The following description will show the method of interpretation of the records secured with this apparatus.

\section{Method.}

Fig. 78 shows the simplest type of a record of speed. This is a record made on the moving strip of paper by drawing a vertical line on the primary sheet directly below the time marker. In order to make this record as simple and exact as possible the vertical guide was put on the hinged plate and the reactor was required to follow the guide in drawing this line.

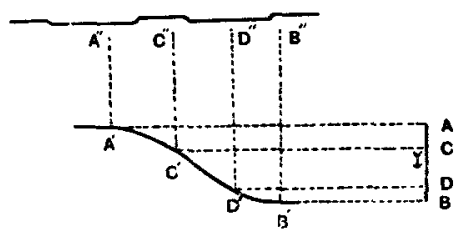

Fig. 78 . (Reduced to one-third.) Illustration of the method of measuring the speed of a vertical line or part of a vertical line which is directly under the time marker. For detailed explanation see text.

The line $A B$ represents the record on the primary sheet and $A^{\prime} B^{\prime}$ and $A^{\prime \prime} B^{\prime \prime}$ the record on the moving strip, $A^{\prime} B^{\prime}$ being the record from the typewriter ribbon, $A^{\prime \prime} B^{\prime \prime}$ the time line. All the dotted lines in Fig. 78 are inserted after the record to aid 
in interpreting the full drawn lines which constitute the record proper. In order to measure the time occupied in drawing the whole line $A B$, we merely have to compare the whole tracing, $A^{\prime} B^{\prime}$, with the corresponding part of the time line, $A^{\prime \prime} B^{\prime \prime}$. The intervals of the time line indicate tenths of a second, and the drawing obviously occupied in this case about $\mathrm{r} \frac{1}{4}$ tenths of a second or $125 \sigma$. If now, instead of measuring the time of the whole line we wish to find the time consumed in drawing any given part of the line shown on the primary sheet of paper, $C D$, Fig. 78 , we need only to project that portion of the vertical line to be measured upon the record taken on the moving strip. The projection of the vertical line upon the oblique record requires that the vertical line and the record be brought into the relation in which they were when the record was made. In order to do this, the reference points on the sheet made by

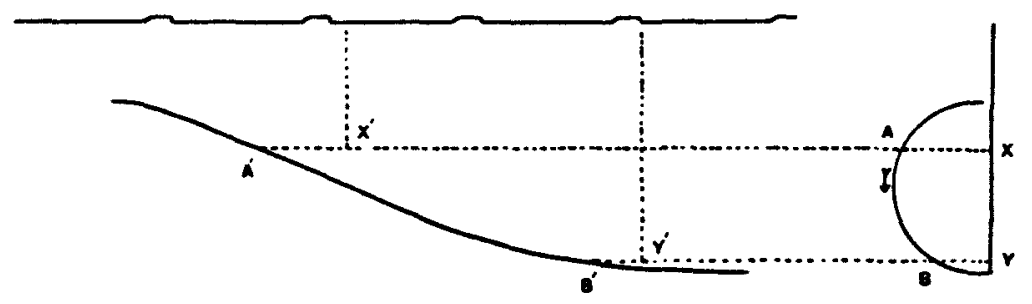

Fic. 79. (Reduced to one-third.) Illustration of the method of measuring the speed of part of a curve. For detailed explanation see text.

the pencils mentioned in describing the apparatus, are superimposed on the reference lines of the strip. Horizontal lines are now drawn from the beginning, $C$, and end, $D$, of the part of the vertical line to be measured, to cut the oblique line of the strip at $C^{\prime} D^{\prime}$. From this stage on the process is the same as in the first case.

The above is the simplest possible case. Very often the line is not drawn directly under the time marker. In such cases a correction must be made, in reading the record on the strip, equal to the horizontal distance between the marker and the line on the primary sheet. In this case the projection of the primary line, $A B$, Fig. 79 , upon the secondary line, $A^{\prime} B^{\prime}$, is found as before. Then the horizontal distance from the 
primary line to the marker, $A X$, is measured, and the same distance taken from the traced line, $A^{\prime} X^{\prime}$. This brings the record of speed under that part of the time line which was made at the same time as the part of the line to be measured. Similar correction is made at $B^{\prime}$ by deducting a distance on the time line equal to $B Y$. The time is now measured on the time line between points vertically above $X^{\prime}$ and $Y^{\prime}$.

This figure shows the principle of correction when it becomes necessary to measure the time of any horizontal or oblique movement. Evidently in all such cases the distance between the two points on the secondary record on the moving strip represents not merely the distance through which the strip travels while the line is being drawn, but also the negative or positive change in horizontal position of the pencil point as it makes the line. The change in position of the pencil point must, therefore, be allowed for either negatively or positively in order to get the simple time determination. In order to make the necessary correction in reading the record, if the pencil moved in the same direction as the strip, this primary pencil movement must be added to the distance between the two corresponding points on the strip; if the pencil moved in the opposite direction, the distance must be subtracted. In the case of an oblique line the horizontal distance through which the pencil traveled must be allowed for, as in the case of the horizontal line. In projecting $A$ and $B$ in Fig. 79 to $A^{\prime}$ and $B^{\prime}$, the distances $A^{\prime} X^{\prime}$ and $B^{\prime} Y^{\prime}$ were recognized as unequal. The greater length of $A^{\prime} X^{\prime}$ shows that the pencil moved obliquely to the right as the line $A B$ was drawn. Hence, a part of the record $A^{\prime} B^{\prime}$ is due not to the movement of the traveling strip, but to the movement of the pencil.

A typical record showing details of a simple reaction is presented in Fig. 8o. $S$ is the record of the reaction signal which is made by the second marker, $M$, Fig. 76 , writing on the moving strip, $B$. In order to bring this signal into relation with the time line a correction has to be made equal to the distance between the two markers. Measuring off this distance on the time line, we find that the signal was given at a point on the time line corresponding to $S^{\prime}$. The point at 
which the reaction occurred is indicated at $R$. The point where the signal is given, thus corrected, will hereafter in all cases be indicated by the letter $S$, and the point of the reaction by $R$.

The error in reading the records was well within three thousandths of a second. Each interval of the marker record, that is, each $100 \sigma$ was in the records $30 \mathrm{~mm}$. or more in length. Accordingly, a distance corresponding to one sigma was at
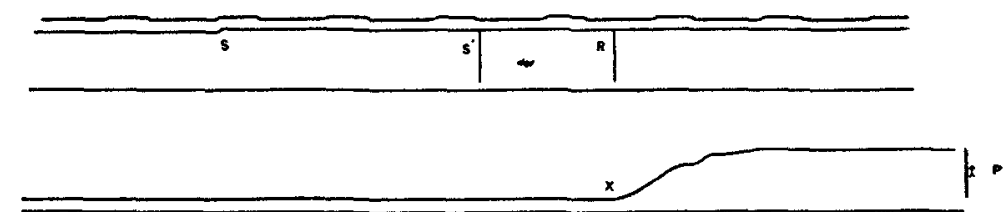

Fig. 8o. (Reduced to one-third.) Speed curve from a reaction by starting a vertical movement upward, showing at $P$ the line as actually drawn, in the upper line the time record, at $S$ the record of the signal for reaction, and $S^{\prime}$ the corrected position of $S$ with reference to the time line. $R$ corresponds to the point $X$ where the reaction began. The reaction, measured by the part of the time line between $S^{\prime}$ and $R$, in this case was very fast, occupying about $125^{\sigma}$.

least one third of a millimeter in length. This could be read with great precision, the measuring apparatus reading directly to tenths of a millimeter. Allowing for all possible errors up to a full millimeter, the error of measurement would fall well within three sigmas.

The reactions reported in this paper consisted of various fundamental movements which occur in all complex writing activities. The reactor held a lead pencil in his hand in the ordinary position for writing and drew lines of various kinds across the primary sheet of paper. The results of this experiment, therefore, constitute an introduction to the study of writing. They also have independent value as showing certain characteristics of various types of reaction movements. In ordinary reaction experiments the movement which constitutes the reaction is very simple, like lifting the finger from the key, and very little is known of the subsequent or antecedent motor changes. Even these simple reactions may very advantageously be subjected to analysis, as has been shown by a number of recent investigations. The present investigation serves to describe a method of complete analysis and to supple- 
ment the analytical work already done on simple reactions by an analysis of more complex reacactions. In the second place the analysis was made complete in two directions in that it includes both horizontal movements and pressure changes. Pressure changes may precede, accompany, or follow the horizontal movement in which the main reaction consists. The secondary phases of movement often throw light on the character of the whole process, as subsequent discussions will make clear.

In the third place, we may by this method study reactions of a type which are ordinarily neglected in reaction experiments, namely, those which consist in stopping a movement. Previous experiments have usually dealt altogether with reactions by starting a movement. We may refer to those common movements as starting reactions. In distinction we may designate reactions by stopping a movement as stopping reactions. In ordinary behavior stopping reactions are as constant and as important as starting reactions. In fact, most reactions are a combination of the two. This is particularly the case with the reactions which occur in writing, as will be seen when one considers a case in which the direction
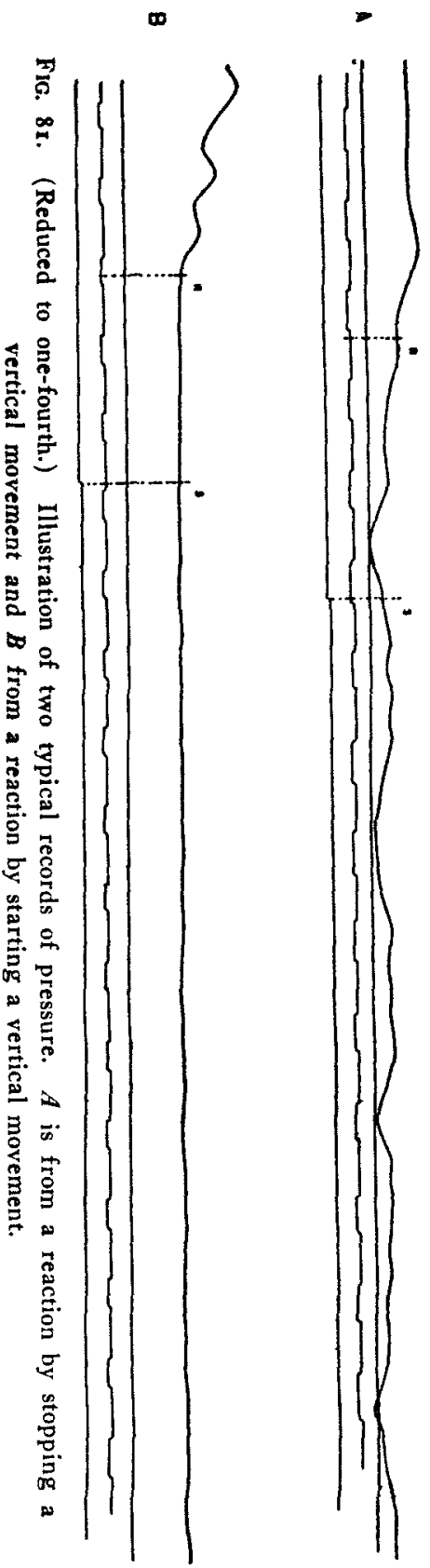
of a line is changed abruptly. 
Since starting reactions are of the more commonly investigated type, we present first a typical case of such reaction. A specimen of the speed record for a starting reaction is shown in Fig. 8o, and the pressure record for the same movement is shown in Fig. $8 \mathrm{I}, B$. In the speed record, Fig. 80, we have the primary line, $P$, and the secondary curve, $X$. The curve consists merely of a straight line before the reaction begins-that is, on the left of $X$. This line is made by holding the pencil at a point, preparatory to the reaction. At the point, $X$, where the line on the record deviates from a straight line, the reaction begins and we may calculate the reaction time by comparing the distance from this point to the point which records the giving of the signal to react, with the time line. From the record after the reaction we can calculate the speed of the post-reaction movement. The more abrupt the deviation of the line from the horizontal, the faster was the movement. Other characteristics come out in the form of the record. In the record before us the movement exhibits marked irregularity in speed, there being two parts of the movement which are much slower than the rest, as shown by the wave form of the line at the right of $X$.

Turning to the pressure curve in Fig. $8 \mathrm{I}, B, S$ is the point at which the signal was given and $R$ the point where the reaction occurred, the record in this case being read from right to left. On the right of $S$ some slight irregularities are seen in the pressure line. These are less obvious in the reduced figure than in the original record. These irregularities show changes in tension prior to the reaction. Similar facts were reported at length in Vol. I. (New Series) of these Studies (pages I4 I184). In this experiment there are shown in addition to the variations in pressure before the reaction, those which occur after it.

The speed and pressure records for a stopping reaction are presented in Figs. 82 and $8 \mathrm{I}, A$. The first part of the speed line, Fig. 82, shows that a vertical movement is being made. At the right end of the record the oblique line changes into a horizontal; this shows the inhibition of the movement. The time which elapsed between the signal to stop and the actual 
stopping can be measured as before. It is especially important to observe that the movement continued for some time after the signal, but is, especially at the end, modified in form. The pressure record, Fig. $8 \mathrm{I}, A$, which should be read from right to left, exhibits the changes in pressure which occur during a vertical movement, and the increase in the downward pressure after the vertical movement has entirely stopped. $S$ and $R$, as before, indicate the giving of the signal and the reaction.

The experiments to be reported fall into seven groups. The details regarding these seven groups are as follows. In Series I. the reaction consisted in stopping a circular movement. The circular attachment was put in the opening of the plate $H$, Fig. 76. The reactor was required to move in an anti-clockwise

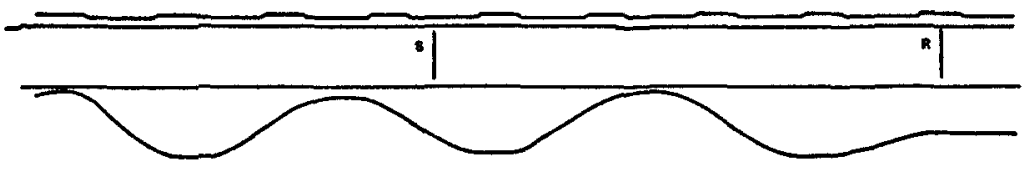

Fic. 82. (Reduced to one-third.) Speed curve from a reaction by stopping a vertical movement, showing in the uppermost line the time record, and in the second line from the top at the extreme left the signal to react. This is corrected to $S$. The line shows at the right of $S$ the break in the circuit of the signal marker, which is not, however, significant. The third line from the top and the bottom line are reference lines. The line between the reference lines shows at the left a succession of upward and downward movements and at the right the stopping of these movements.

direction, keeping the point of the pencil against the inner edge of the guide. The movement was made at a rate of speed which was agreeable to the reactor, and varied from one to two revolutions a second. The reaction consisted in stopping the movement as abruptly as possible when the sound was heard.

In Series II. the reaction consisted in starting the circular movement which was used in Series I. The pencil was held at a point against the inner edge of the circular guide and at the signal the reactor began to trace a complete circle. In one half the reactions the point at which the movement began was at the extreme left side of the circle, and in the other half at the extreme right side. 
In Series III. the reaction consisted in stopping a vertical movement. The vertical guide was put in the opening of the hinged plate. The reactor moved the pencil up and down against this vertical guide, tracing a line on the paper. The movements were made at the rate of about two double strokes per second and were from two to three centimeters in extent.

In Series IV. the reaction consisted in starting the movement described in Series III. The reactor held the pencil at a fixed point against the straight edge and at the signal made a single stroke. In half the cases the movement was made from the top down, and in the other half from the bottom up.

In Series V. the same vertical movement as in Series III. was made before the reaction. The reaction itself, however, consisted in changing the direction of the movement from the

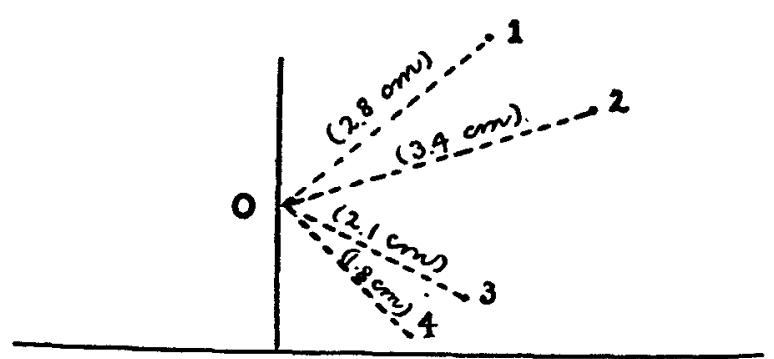

Fig. 83. (Natural size.) Position of the points used in the reactions in series VI which consisted in moving to points. $O$ is the point on the straightedge at which the pencil was held preparatory to the movement in each reaction.

vertical to the horizontal, instead of merely stopping it. At the signal the reactor moved the pencil from the vertical, whatever its position happened to be, through a convenient distance horizontally to the right.

In Series VI. the reaction consisted in moving from a fixed point, $O$, Fig. 83 , which was directly under the time marker, to a dot which was placed on the paper. Four dots were used for successive trials in the positions and at the distances indicated in Fig. 83. The reactor was instructed to move to the point with one stroke.

In Series VII. the reaction consisted in starting to make a square or a circle. The pencil was held before the reaction 
directly under the marker, as in Series VI. The square or circle as made are shown in Fig. 84. In half the cases the reactor started up and in the other half down.

In most of the reactions of all the series a warning signal
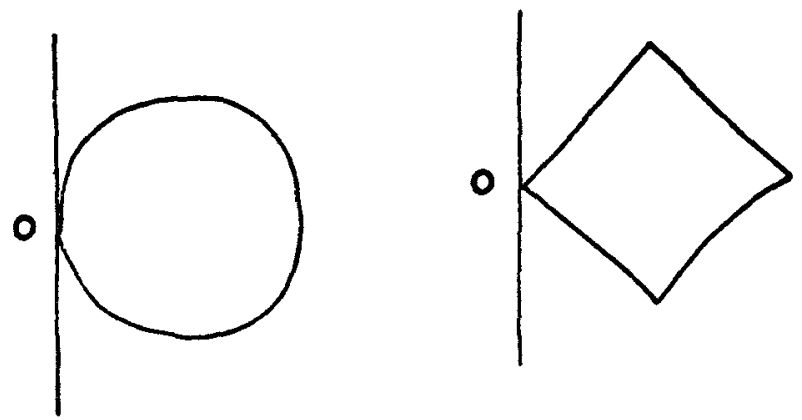

Fig. 84. (Natural size.) Types of the figures made in the reactions by starting to make geometrical figures.

was given by saying ' ready' from one to two seconds before the signal for reaction.

There were five subjects in the experiment, all of whom were trained in reaction work. They were Chas. H. Judd, E. H. Cameron, D. J. Cowling, C. A. Cockayne and the writer. They will hereafter be referred to by their initials.

\section{RESULTS.}

The results of the experiment fall under three general heads. First, the reaction times of the various series will be reported in thousandths of a second. Second, a description will be given of the form of the speed curve and the rate of its different parts. Third, a report will be given of the pressure changes which occur before the signal, between the signal and the reaction, and after the reaction.

The reaction times obtained in each of the series of the experiment are shown in Table I. This table gives the number of reactions, the average reaction time, and the mean variations for each reactor in each series. The total number of reactions and general average is also given for each series.

The mean variations are in some cases within ten per cent. of the average, but are usually larger than ten per cent. and in 
TABLE I.

Reaction Times of the Different Subjects under the VARIOUS CONDITIONS.

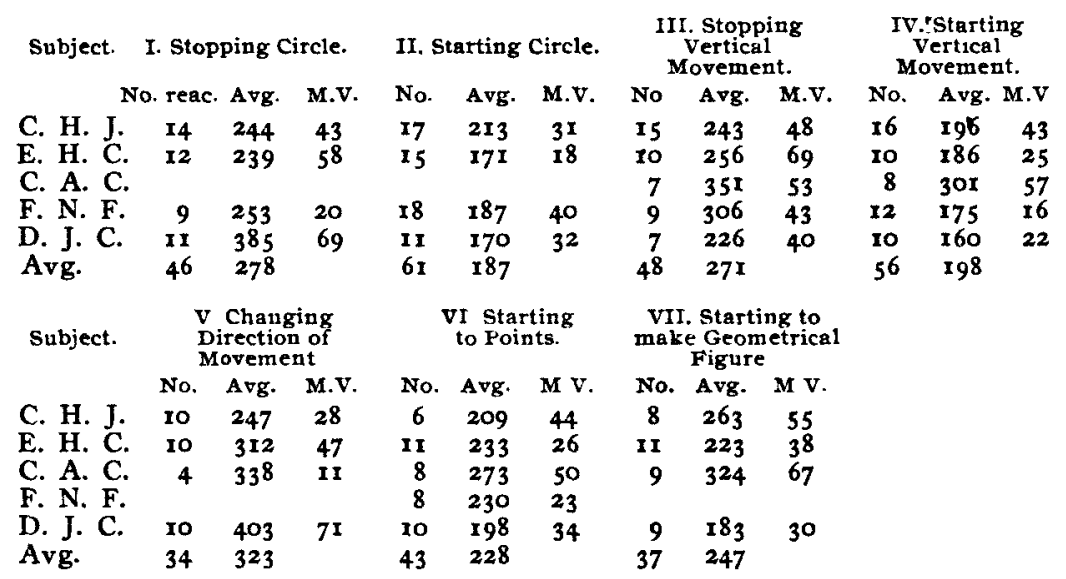

several cases are about twenty per cent. The explanation of this large mean variation is probably to be found in the complexity of conditions which were imposed on the reactors.

A comparison of the stopping reactions with the starting reactions shows that the stopping reactions are markedly slower. Take first Series III., stopping a vertical movement, and Series IV., starting a vertical movement. The average of the stopping reactions is longer by $73 \sigma$ or 37 per cent. than the average of the starting reactions, and the average for each subject shows a like difference. The same general result appears in the starting and stopping of circular movements. In dealing with the vertical movements, it was thought that possibly the pause and abrupt changes in direction in the vertical movements prior to the particular movement in which the stopping occurred, might be the cause of the delay in stopping when the signal was given, and the circle was accordingly introduced to secure a movement without pauses or abrupt changes in direction. By comparing the results of Series $\mathrm{I}$. and II., it will be seen that there is a still greater difference between the time of stopping and starting a circular movement, viz., 9 I $\sigma$ or 49 per cent. The stopping reactions are slower than the starting reactions in this case also for each reactor. 
The averages of the two other starting series, Series VI., starting to points, and Series VII., starting to make a geometrical figure, are also, in spite of the complexity of the conditions in these series, for the most part smaller than the averages of the stopping reactions. This rule holds in the case of the individual reactors with but one exception. For C. H. J., Series VII., starting to make geometrical figures was slower than the stopping reactions.

Turning to a comparison of all the starting reactions, viz., Series II., starting a circle; Series IV., starting a vertical movement; Series VI., starting to points; and Series VII., starting to make geometrical figures; it is seen that the last two series, which involve complex preparation, are noticeably longer in most cases than the first two. This result holds for the individual reactors with two exceptions; with $\mathrm{C}$. $\mathrm{H}$. J., starting to points is faster than starting a movement in a circle; and with C. A. C., starting to points is faster than starting a vertical movement. These are but two exceptions out of eighteen cases. It appears, then, that a reaction in which the succeeding movement has a greater number of determining conditions is slower even in starting than a reaction in which the movement has fewer conditions. In starting a movement to a point the reactor has not only to make a movement in a specified direction, as in starting a movement in a circle or along a straight edge, but he has also to stop the movement at a given point. In starting to make a geometrical figure, he has to move through a given distance and then change the direction of the movement. The later stages of the movement are evidently prepared for before the actual turn or pause is made, so that the earlier phases of the reaction are modified in view of the later requirements. Changing the direction of a movement, Series V., has the longest reaction time of all with two individual exceptions. For C. H. J., Series VII., starting to make geometrical figures, is longer, and for C. A. C., Series VII., stopping a vertical movement is longer. This reaction, as will be pointed out in the later analysis, is the most complex of all.

Turning from a consideration of the reaction times to the 
character of the records, it will be found convenient to consider separately three parts of the records, namely: first, the parts before the signal for reaction; second, the parts between the signal and the reaction; and, third, the parts after the reaction.

The traced records before the signal are of interest only in the stopping reactions, viz., in Series I. and III., and in the
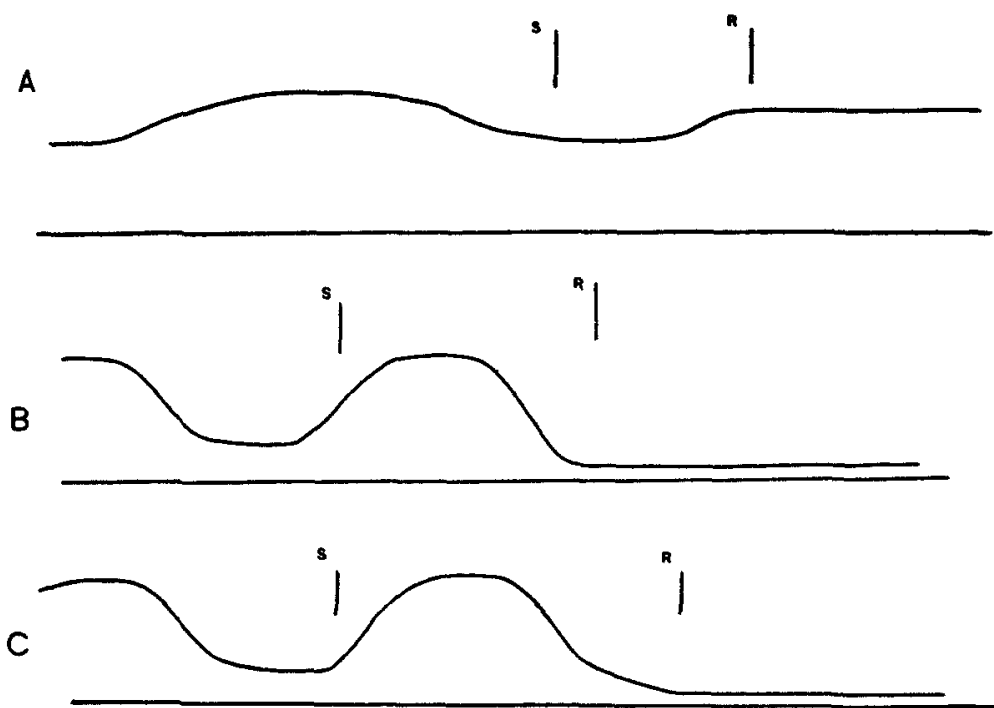

Fig. 85. (Reduced to one-third.) Speed curves from reactions by stopping a vertical movement. $S$ indicates the point where the signal is given and $R$ where the reaction occurs. $A$ shows increase in speed of upward movement between the signal and reaction, $B$ shows increase in the speed and amplitude of the movement and $C$ shows decrease in the speed and increase in the amplitude.

records of changes in the direction of movement, in Series $\mathrm{V}$. In the other series the traced record before the reaction is merely a straight line. Fig. 85 gives three records from Series III., and Fig. 86 gives five records from Series V. All of these curves are records which can be used in determining the speed of straight line movements. In all cases the speed variations before the signal to react are fairly regular. In the case of vertical movements the speed varies from a maximum at the middle of each stroke to a minimum toward each end, with a final pause at the end of each stroke. 
Series I. gave records of circular movements prior to the reaction. In order to ascertain the relative speed of different parts of circular movements, measurements of time required for equal arcs of upward and downward circular movements were made. The results of these measurements are interesting on account of their bearing on writing. Table II. gives the average time of the upward and downward strokes, the difference between them and the number of cases in which the downward strokes and the upward strokes, respectively, were slower. It will be seen that although each person shows a rather uniform relation between the speed of the upward and downward strokes, no two of the subjects of the experiment belong to the same type. Measurements would have to be taken from a large number of subjects in order to determine what is the most common type and what are the causes of the difference between different individuals.

TABLE II.

Comparison of Upward and Downward Strokes in Making Circles.

\begin{tabular}{|c|c|c|c|c|c|c|}
\hline Subject. & $\begin{array}{l}\text { No of } \\
\text { Cases. }\end{array}$ & $\begin{array}{l}\text { Average } \\
\text { Downward } \\
\text { Sirokes } \\
\text { Sigmas }\end{array}$ & $\begin{array}{l}\text { Average } \\
\text { Upward } \\
\text { Strokes. } \\
\text { sigmas. }\end{array}$ & Difference. & $\begin{array}{l}\text { No. of Cases in } \\
\text { Which Downward } \\
\text { strokes Were } \\
\text { slower. }\end{array}$ & $\begin{array}{c}\text { No. of Cases in } \\
\text { Which Upward } \\
\text { Strokes were } \\
\text { Slower. }\end{array}$ \\
\hline C.H. $\mathrm{J}$. & 15 & 1,509 & 1,435 & -74 & I 3 & 2 \\
\hline F. N. F. & 16 & 1,407 & 1,395 & - I2 & II & 5 \\
\hline D. J.C. & I I & 998 & 1,003 & +5 & 5 & 6 \\
\hline E. H. C. & I3 & 1,409 & $x, 549$ & +140 & I & I2 \\
\hline
\end{tabular}

No new observation regarding the changes of rate of movements before the reaction need to be added in view of the report given for Series III. and V.

Turning now to the second part of the traced record, that lying between the signal to react and the final movement of reaction, we are again confined to Series I., III. and V. A number of typical cases are presented in Figs. 85 and 86 . In some cases the speed of the movement is increased, as in $A$ and $B$, Fig. 85 , and $D$, Fig. 86 . The record line here falls or rises more abruptly than in the preceding part of the record. Conversely, a larger number of cases show that the rate of a preliminary movement is gradually reduced as the moment of reaction approaches, indicating that the usual method of stopping a movement was to 'slow down' gradually to a stop. This change is seen in $C$, Fig. 85, and $C$, Fig. 86. This would 
seem to be the natural method of stopping. Where there is an abrupt acceleration of speed before reaction followed by a sudden stopping, we have a result analogous to the antagonistic reaction noted by Smith in Mind, Vol. XII., pp. 47-58, and in Vol. I. (New Series) of these Studies, pp. I41-I84.

Changes in the amplitude of the movement can obviously not occur when the circular guide is used, but only with the straight edge, in stopping or changing the direction of a vertical movement, Series III. and V. Such changes between the signal and the reaction are shown in curves $B$ and $C$, Fig. 85 , and $C$, Fig. 86. Decrease in amplitude is shown by the fact that the upper part of the tracing is lower or the bottom higher than in the preceding part of the line, so that the vertical distance between them is less. Change in amplitude almost always consists in a decrease of the amplitude, indicating that in this respect, as in speed, the movement comes to a stop gradually.

In certain cases of vertical movement there is an increase in the length of the pause at the beginning of the last preliminary movement. There is always a pause, as was noted above, at the top and bottom of each vertical movement. When this pause is lengthened before the last preliminary movement it indicates clearly a conflict of tendencies. The movement is well established and there is a strong tendency to continue it. The signal calls for a stopping of the movement and is only slowly and partially successful in bringing about its result. The lengthening of these pauses between the signal and the reaction occurs chiefly in changing the direction of a movement, Series V., which is evidently a complex form of reaction, as shown by the time given in Table I. Such a lengthening is clearly shown in curves $A, B$ and $D$, Fig. 86 . This increase in time of the reaction and change in the form of the movement shows that what usually occurred was that the horizontal movement was not continuous with the vertical movement, but the vertical movement was first stopped and then the horizontal movement was begun. There are thirtythree reactions in which the above-mentioned changes in the character of the movement occur between the signal and the 
reaction. In some reactions more than one change is present, as, for example, a decrease in the amplitude and a decrease in the speed of the movement.

Significant traced records after the reaction appear chiefly in Series V. The character of the primary line in Series V.,
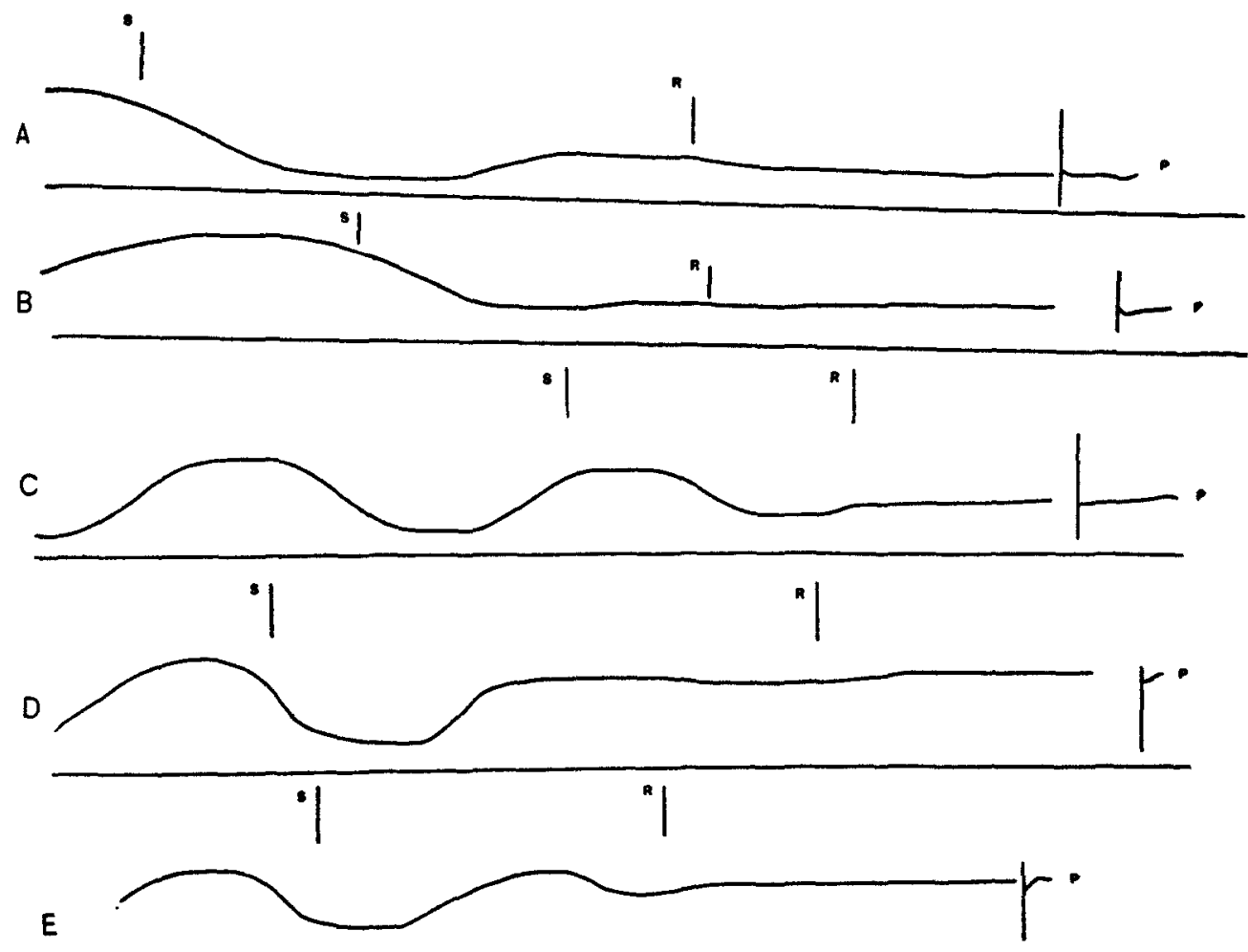

Fig. 86. (Reduced to one-third.) Speed curve from reactions which consisted in changing the direction of a movement, and the corresponding lines as actually drawn on the primary sheet. The latter are indicated by the letter $P, P \ldots A$ shows a decrease in the speed of the vertical movement, a pause before the initiation of the horizontal movement and an inclination of the horizontal movement downward toward the movement just preceding it. $B$ shows a pause before the reaction and an inclination of the horizontal movement away from the movement just preceding it. $C$ shows a decrease in the speed and amplitude of the vertical movement before the reaction and an unusually straight horizontal primary line. $D$ shows marked increase in speed before the reaction and a pronounced pause between the signal and the reaction. $E$ shows an inclination of the horizontal movement toward the downward movement just preceding it. 
which was made by changing the direction of a movement, is worthy of detailed examination. Examples of these lines are shown in Fig. 86 and are indicated by the letter $P$. These are the primary lines of which the long curves are the secondary lines. The general fact is that the apex of the angle which the horizontal line forms with the vertical line is very much rounded and the first part of the horizontal line is a pronounced curve. $A, B$ and $E$, Fig. 86 , are examples of two types of these lines. In $B$ the concave side of the curve faces the last stroke before the horizontal movement. This is the more usual type. In $A$ and $E$ the curve is in the opposite direction. This form occurs in a number of cases. $C$ is an unusually close approximation to a horizontal line. The straight horizontal movement, then, is not attained immediately, but there is a vertical component at first, which disappears gradually. The variations in form show how difficult it is to secure any. thing like a separate and wholly new movement immediately. Here the established vertical movement tends to pass over gradually into the new movement. In contrast with the earlier cases in which it was shown that preparation for a later movement reaches back into the early stages of a reaction, the influence here is forward. Though the two types of cases differ in the direction of influence, they agree in the general fact that there is a tendency for any single reaction to reflect in its character and rate the surrounding condition in which it occurs. The traced curves show also the transition to the new movement.

We come now to a consideration of the pressure records. A pressure record was taken for each subject, but as it was not convenient to prepare more than one strip of smoked paper for each period, only two or three pressure records on the average were taken with each series. Since the characteristics of the pressure curves were fairly uniform for each kind of reaction, however, and since they were used only for qualitative determinations, this number of records is sufficient as a basis for the description of pressure changes. There are 59 pressure records in all, distributed among the series as follows: Series I., stopping a movement in a circle, 6 ; Series II., starting a move- 
ment in a circle, 7 ; Series III., stopping a vertical movement, I8; Series IV., starting a vertical movement, 7; Series V., changing the direction of a movement, 6; Series VI., starting to points, 6; Series VII., starting to make geometrical fig. ures, 9 .

Fig. $8 \mathrm{I}$ shows two typical records, $A$ from D. J. C. taken from Series III., stopping a vertical movement; and $B$ from C. A. C. taken from Series IV., starting a vertical movement down. The lower line in both records is from the signal marker and the break indicates the signal to react. The second line from the bottom is the time line marking tenths of seconds. The third line is the straight line traced as a base for comparison with the pressure curve. The fourth line is the pressure record. As $B$ is the simpler record, we will first analyze that. There first appears before the signal a slightly wavy line, whose crests are from $50 \sigma$ to Ioo $\sigma$ apart. The pencil was here held at the fixed point before the reaction with considerable pressure and with slight oscillations in pressure, these oscillations being of a tenth of a second or less in duration. These waves can be satisfactorily explained as due to the succession of nervous impulses necessary to hold the hand in a fixed position. Between the signal and the reaction the waves are less prominent, indicating a change in tension preparatory to the reaction. Just before the reaction shown on the traced record, the pressure begins to increase gradually, and finally, soon after the reaction, which consists in beginning a movement along the straight edge, it increases rapidly by three stages. This marked rise in pressure after the reaction is a feature which appears in nearly all of the reactions. Indeed, the examination of such a record as this gives one a very striking example of the complexity of a reaction. The drawing of a vertical line is evidently accompanied by a series of pressures against the writing surface which must be important in getting the muscular mechanism and the nervous mechanism into operation, but all of which are neglected in any ordinary record of action.

Record $A$, Fig. $8 \mathrm{I}$, shows the typical variations in pressure during the making of a series of up and down vertical move- 
ments. There are minor variations among different subjects, but the general characteristics for such movements are alike. For the same subject, as will be seen from this record, successive movements of the same kind are accompanied by practically the same pressure variations. In the present case a detailed comparison of the pressure record with the speed record shows that for a straight up and down movement the point of least pressure is about two thirds of the way on the upward stroke. The pressure then increases as the stroke approaches the top, continues to increase, sometimes after a slight decrease, as the stroke comes down and reaches its maximum intensity half to three quarters of the way on the downward stroke. It then decreases and reaches its lowest point again a little past the middle of the upward stroke. A modification of this series which frequently occurs consists of a small secondary increase in pressure at the beginning of each stroke.

The other significant facts shown in record $A$, Fig. $8 \mathrm{I}$, are the changes in the pressure variations between the signal and the reaction and the great increase in pressure after the reaction. The signal for reaction comes when the upward stroke is about one third completed. The pressure decreases as usual to its minimum as the stroke goes up, increases as the stroke approaches the top and after a slight decrease increases as the stroke goes down. The pressure changes are as usual up to this point. Here, however, instead of decreasing as the stroke reaches the bottom, it increases rapidly. It remains constant till a short time after the reaction and then increases to about double its previous greatest intensity. This pressure is then maintained. This shows a notable pressure reaction before the primary reaction.

A number of curves showing other examples of pressure changes with some variation are presented in Fig. 87. Record A, from C. A. C., is one of the several cases in which an effect of the warning signal in a change of pressure, without an actual premature reaction, is evident. The fall of the pressure curve below the base line near the right-hand end of the curve shows a partial reaction to the warning signal by a change of pressure, but there was no movement of the pencil across the 
PRELIMINARY EXPERIMENTS ON WRITING REACTIONS. 325

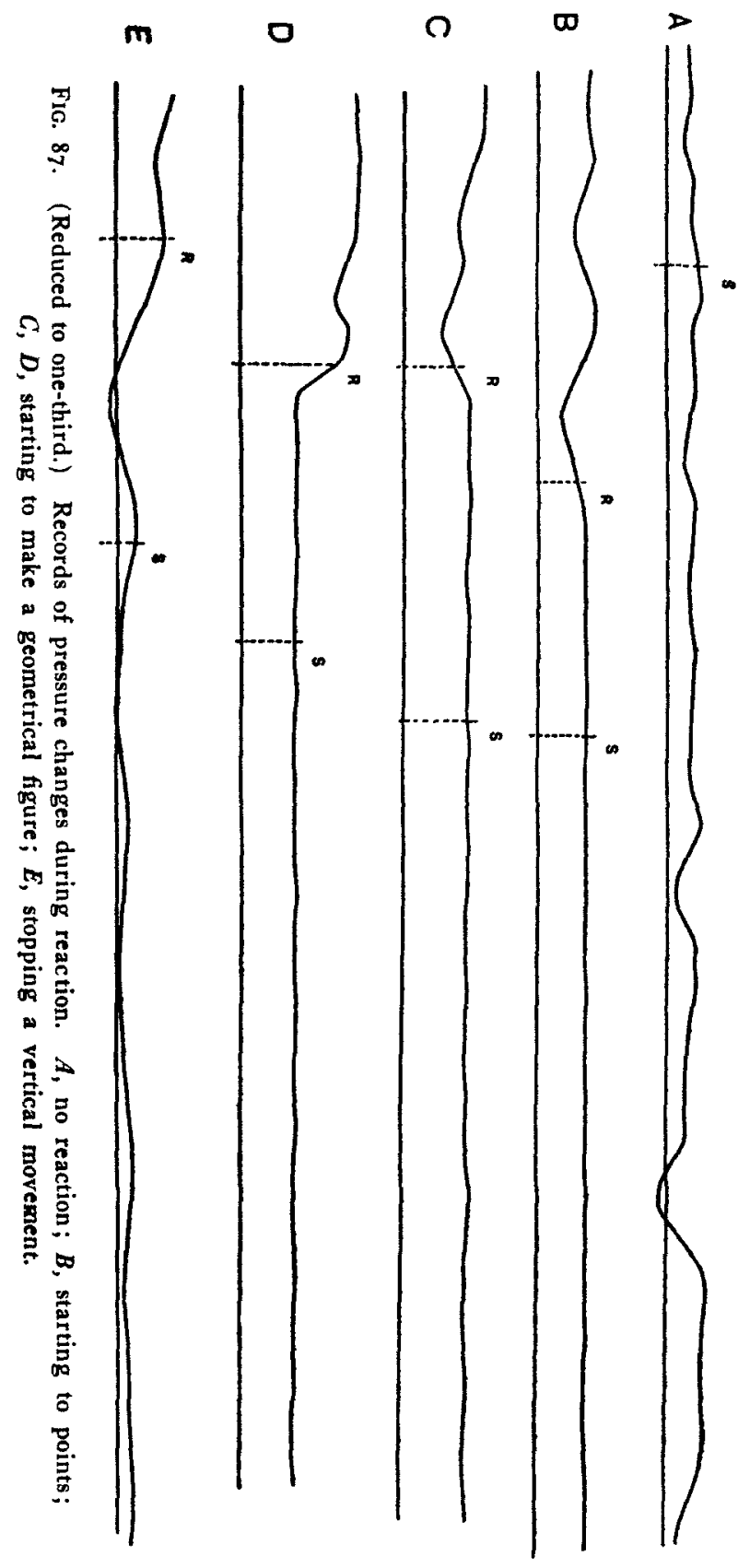


paper either here or after the reaction signal. In some cases there was also an actual premature reaction to warning, both of pressure and movement across the paper.

Curve $B$, from C. A. C., is from the pressure record of a reaction in Series VI., starting to points. This record shows a slight waviness of the pressure line before the reaction. About $35 \sigma$ before the true reaction there is a pronounced decrease in the pressure. After the reaction the pressure continues to decrease, then increases and oscillates while the reaction line is being drawn. Here again the pressure reaction and the reaction proper are seen to be different phases of the total reaction.

$C$ is from E. H. C., in Series VII., starting to make a square. The waviness of the pressure line while the pencil is held at a fixed point before the reaction is here well marked, and continues up to the moment of the reaction. The pressure decreases before the reaction as in $B$, and increases somewhat more after the reaction.

A comparison of the average height of curves $B$ and $C$ indicates a typical difference in the amount of pressure exerted by different individuals. Pressure also varies somewhat with the same individual at different times.

$D$ is from the same subject and series as $C$. It has the same characteristics until just before the reaction. In this reaction the pressure increases instead of diminishing at the reaction. The increase of pressure which accompanies the drawing of this line begins, as in the previous case, before the reaction. The intensity of pressure after the reaction is greater in this case.

$E$ is from C. H. J., in Series III., stopping a vertical movement. It exhibits the typical pressure variations accompanying the vertical movements. The reaction comes at a point about two thirds down the stroke. The pressure ordinarily rises in this part of the record to its maximum intensity, but in this case the rise in the pressure is directly related to the reaction, as is shown by the fact that it is more than double that of any previous stroke. This is also another example of a pressure change coming before the reaction. After the reac- 
PRELIMINARY EXPERIMENTS ON WRITING REACTIONS. 327

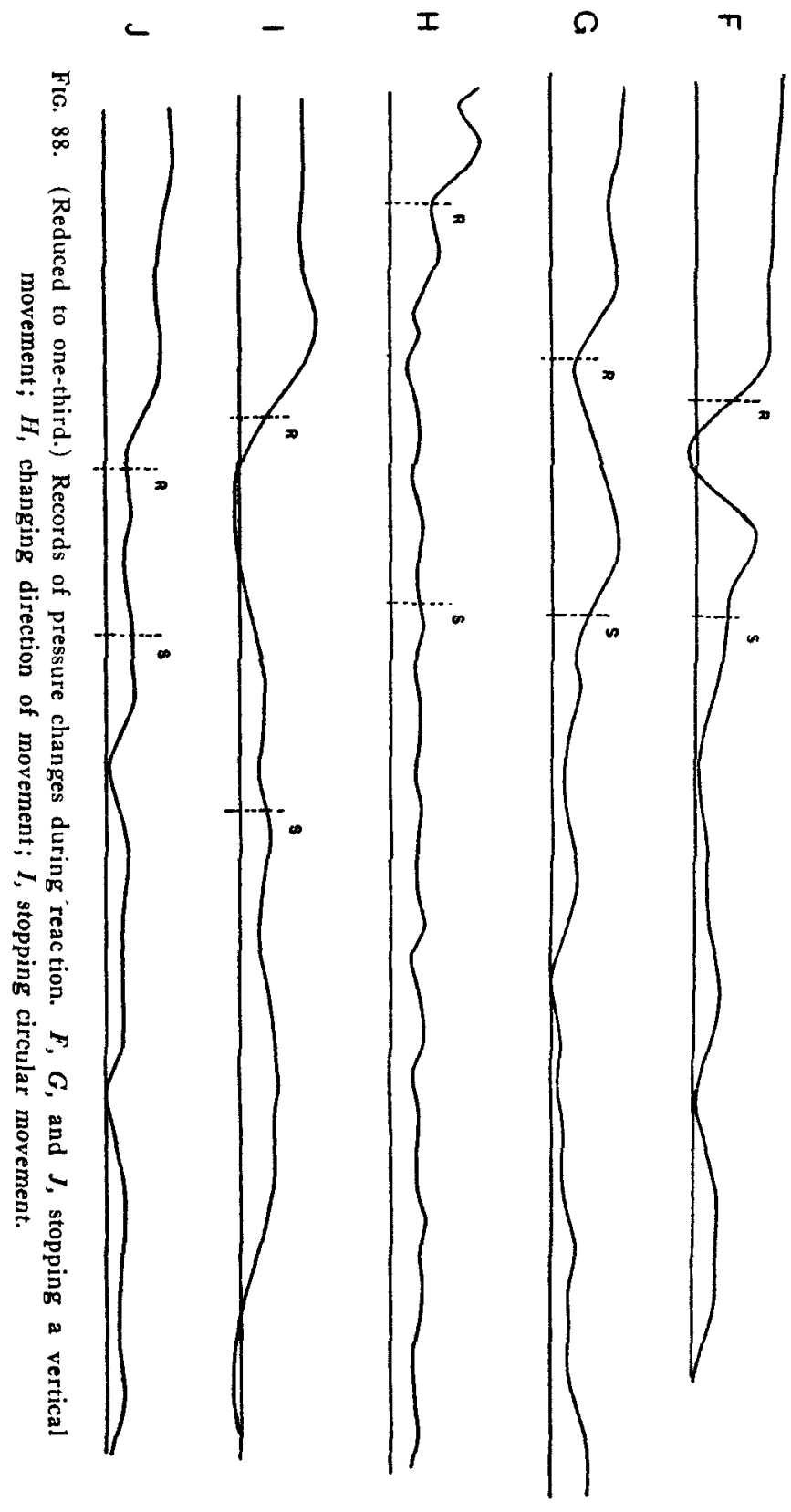


tion, the pressure decreases slightly and then increases again as usual. While the pencil is held at a fixed point after the reaction the pressure is not so steady as it is when the pencil is held at a fixed point under ordinary circumstances. It oscillates with a rhythm similar to the pressure variations which accompanied the movement before the reaction.

Fig. I 3 shows similar pressure curves.

$F$, from the same reactor and series as $E$, is typical of certain cases in which a pressure change similar to those which are described above occur before the reaction comes and within IOO $\sigma$ of the signal to react. The pressure reaction time is accordingly as short as the most rapid forms, while the apparent time measured from the primary movement is slower than the usual reaction.

$G$ is another record from the same reactor and series showing the same sort of pressure change coming about roo $\sigma$ after the signal. These records show that there may be a response to a signal in less than $100 \sigma$.

$H$ is from C. A. C., Series V., changing the direction of a movement. This record shows especially regular pressure variations for the preparatory movement. This movementthe vertical movement-stops a little over half way up the stroke. The pressure here increases with a little jerk, producing the curve on the record about two thirds of the way from the signal to the reaction. The hand then pauses before beginning the horizontal movement which constitutes the reaction. Meanwhile the pressure increases rapidly until the reaction. This is another case in which the increase in pressure comes before the movement which it ordinarily accompanies and which constitutes the reaction. The movement which was intended as a horizontal movement is of the second type described above ( $E$, Fig. 87 ), its convex side faces toward the movement last made. That is, it turns back on the last stroke. The pressure increases rapidly as this movement is made.

$I$ is from F. N. F., Series I., stopping a circular movement. The extremely low pressure comes in the fourth quadrant of the circle. In this quadrant the pencil twice left the paper. The reaction occurs about half way on the upward movement, 
that is, at the extreme right-hand side of the circle. The pressure increases as usual up to this point and when the pencil ceases to move at the reaction, the pressure continues to increase rapidly till it reaches a high level, which is maintained.

$J$, from C. H. J., in Series III., stopping a vertical movement, shows the usual rise in pressure after the reaction, and a continued oscillation, suggesting, as in the record $E$, the continuance of the pressure changes which accompany the previous movement.

The characteristics which have been pointed out in these records are typical of the pressure changes accompanying the reactions. For example, out of the twenty-nine records in which the pencil was held at a fixed point before the reaction, fifteen showed a waviness in the pressure curve. Of all the records in which a reaction took place, thirty showed a noticeable change in pressure between the signal and the reaction. In twenty-five cases the pressure increased and in five it decreased. In all cases there is marked change in pressure after the reaction, usually a very pronounced increase. In half of the cases in which a movement is followed by holding the pencil at a fixed point, that is, in eleven out of twenty-two, there is a noticeable oscillation in the pressure line while the pencil is thus held, suggesting a continuation of the previous pressure variations.

\section{Discussion.}

In the discussion of the results of this experiment it will be necessary to abandon the distinctions which, for the sake of clearness in description, have been drawn between the various phases of the reaction. No one of the elements, as for example the reaction time, can be considered alone. The difference in the time of various reactions must clearly include some reference to the complex of activity which makes up the reaction. To separate the duration from the other characteristics is to make an arbitrary distinction which obscures rather than aids in the study of the problem.

The point of departure for the explanatory discussion may be taken from the fact first noticed, namely, that the stopping 
reactions were slower than the starting reactions. Before generalizing on the results it should perhaps be noted that neither the movements in a straight line nor in a circle, as arranged with the apparatus described, were continuous in a single direction. It is very desirable that measurements of stopping and starting reactions be taken with long movements in a single line. It may be said, however, that the forms of movement used in the experiment are more closely analogous to the great majority of the movements which one makes in every-day life than the simpler form would be. That is, most movements, as in walking, writing, or manipulation of any kind, and also the involuntary activities, involve frequent and more or less regular readjustment in direction. The results here reported would, therefore, apply to these movements.

A movement of the kind under consideration, then, involves not only a continual change in position but also a frequent, and in the case of the circular movement, a continual readjustment of direction. The character of the innervation, the muscular coordination and the direction of application of energy is ever changing. The inhibitory impulse and the type of muscular activity necessary to stop the movement must be different for every change in the movement. Stopping a movement is not a passive affair nor is it a uniform inhibitory process which may be imposed alike on the movement at any point. It is rather an active process, the precise character of which is determined by the particular form of movement to be stopped. It involves a withdrawal of innervation from the muscles which produce the movement and an innervation in turn of the antagonistic muscles. From the point of view either of consciousness or of the physiological mechanism, then, no preparation of a general sort can be made for stopping a movement, such as that involved in the experiment. The attention can not be directed to any point in the movement nor to any specific motor adjustment. No muscles can be innervated nor any nervous path 'set' in preparation for the stopping.

With the starting reaction, however, the case is different. The process of preparation for a reaction by a partial innervation of the muscles which produce the movement and a 'set' 
of the nervous path is too familiar to need more than mention. It is easy to understand from the greater complexity of the stopping reactions and the fact that they permit of no preparation why they require more time than the starting reactions.

The qualitative records of these reactions bear out this statement of the difference between the starting and stopping reactions. The first response to the signal in stopping a movement is a diffuse spreading of the innervation, rather than a precise reaction along well-defined paths. This causes in the majority of cases at the same time a decrease in the speed and amplitude of the movement which is being made and an increase in the pressure exerted. In contrast to this, in the starting reactions a decrease in pressure often came at first, showing that the innervation is applied directly to the muscles involved in the precise reactions. When the movement was stopped the energy was not withdrawn but was transferred by an elaborate readjustment to another set of muscles.

If the explanation offered of the difference in the time of stopping and starting a movement is correct, we should expect that changing the direction of a movement would also be slow, for changing the direction of a movement consists in some cases, as was pointed out in the description of the records, in first stopping the movement and then beginning a new one in a different direction. The complete process already described as characteristic of stopping a movement had to take place and an additional activity had to be inaugurated to produce the new line. The case is further complicated by the fact that the particular activity which is required to produce this new movement is dependent upon the point in the earlier movement at which reaction happens to take place. Sometimes, it is true, the reactor seems to be able to eliminate the stopping process to a certain extent, and make the horizontal movement continuous with the vertical, without a pause; but the effort is usually only partly successful, as shown by the fact that the new movement starts not horizontally, but at an acute angle from the vertical line, indicating that the innervation into the old channels was not entirely inhibited before the new impulse began to act. In any case this process would be as complex as that of stopping 
a movement, the coordination producing the new movement being substituted for the coordination which produced the inhibition of the movement.

The results in all these cases of movements turned into a new direction should be recognized as results with reactors who were not especially expert in the particular kind of movement here undertaken. If the explanations are to be applied to longpracticed activities, such as those of writing, it should be recognized that the succession of innervations is much less delayed after practice. There is even then a necessity of stopping the old reaction and beginning a new reaction, but the well-trained coordinations show little of the diffuse period. Indeed there were some cases of reaction by changing the direction of a movement in which the diffuse period was much shorter than usual, but it was nearly always long enough to indicate that the reaction was more complex than a simple stopping reaction. Among the results reported in this paper the descriptions given of the transitions from one part of a circle to another, or from an upward line to a downward line are better examples of welltrained and fully prepared transitions in movement.

The greater length of the reactions in drawing a line to a point or in making a geometrical figure would seem to be susceptible of a similar explanation. In these cases the initial movement was definite enough, but the attention was divided between the initial movement and the later stopping of the movement. The movement was not only to be made, it was also to be stopped or its direction changed at a certain place. This fact must have resulted not only in the innervation of the muscles employed in the initial movement, but also in the partial innervation of the muscles used in the subsequent stopping or change in direction, and this must have interfered with the rapidity of the reaction.

The analysis of movements undertaken in this investigation shows that there are a great variety of readjustments which are not fully and explicitly recognized by the reactor. This can be brought out by reporting certain introspections which the reactors gave during the series. For example, one reactor introspected the vertical starting movements, 
especially the upward movements, as slower than the stopping movements. On the contrary, the starting movements were the faster and of these the upward movements were the faster. Again a reaction was sometimes introspected as slow when the reaction itself was fast, but the movement following the reaction was slow. One reactor, for example, said that, in Series III., stopping a vertical movement, he always stopped before the stroke was finished which he was making when the signal came, provided the signal came near the beginning of the stroke. If the signal came past the first part of the stroke he said he stopped within the next stroke. As a matter of fact he in no case stopped before he had made two strokes after the signal.

Again, the pressure changes were not recognized as pressure changes. Though they were constantly present the reactors were for the most part wholly unaware of them; they existed for the reactor's consciousness not as pressure changes but as changes in the position and character of the movement.

This confusion of the factors involved in movement is explained if we regard the function of consciousness as not primarily to mirror the separate elements of movement, but to grasp it as a unit, though a progressively changing one.

The reactor was not conscious of changes in the speed and amplitude of movement, or of the changes in intensity of pressure which have been described as occurring between the signal and the reaction. These were merged for consciousness into the perception of the reaction. The changes in speed and amplitude and the final stopping made up the change of balance between rest and motion which constituted the reaction, but only the total change and not the gradations from motion to rest were apprehended.

The results of this investigation emphasize the unity of a reaction process and emphasize further the complete parallelism between the conscious attitude as a whole to the reaction complex as a whole, while showing clearly that consciousness does not reflect in detail the factors of the reaction. 